\title{
CHIO DEI GENOVESI TRA RIVOLTA MAONESE, CORSARI CATALANI ED ATTACCHI VENEZIANI
}

\author{
LAURA BALleTto \\ Universitd di Genova (Italia)
}

La cronaca dei fratelli Giorgio e Giovanni Stella è certamente uno dei testi più importanti nella cronachistica genovese fra il secolo XIV ed il XV. Giovanni Stella fu "uomo di governo e uomo di lettere, uno dei tanti cancellieri-letterati dell'epoca, contemporaneo e collega di Iacopo Bracelli, di Biagio Assereto e dello stesso fratello Giorgio: i grandi nomi della cancelleria genovese, che dettero vita al primo importante cenacolo letterario sorto a Genova"1. La sua cronaca, che comprende gli avvenimenti fra il 1406 ed il 1435, e la continuazione di quella del fratello Giorgio, che giunge fino al 1405. Fu continuata poi fino al 1461 da Battista Stella, figlio di Giorgio: ma questa parte non ci è pervenuta ${ }^{2}$.

Oltre ad attività pubblica, Giovanni sviluppò anche attività letteraria. Ancora in giovane età, nel 1392 (era nato intorno al 1370), invio a Coluccio Salutati un suo componimento poetico in cui esaltava la pace conclusa tra Firenze e Milano grazie alla mediazione del doge genovese Antoniotto Adorno. Sono opera sua gli otto versi commemorativi incisi sul sepolcro di Giovanni Doria (+1401) nella chiesa di Santo Stefano di Genova, come pure i versi composti per ricordo dell'ampliamento di Castelletto ed incisi sulla porta d'ingresso a lode del Boucicaut, governatore francese di Genova, i quali recitano:

\footnotetext{
'Georgii et Iohannis STELLAE, Annales Genuenses, a cura di Giovanna PETTI BALBI, Bologna, 1975, p. V (ed ivi bibliografia citata).

'Ibidem, p. VI.

"Anuario de Entudios Medievales". 24 (1994)
} 
Francorum regis titulus et iura reservans, Arx excelsa loco tibi, Ianua, presidet isto, Mille quadringentis uno currentibus annis, Condita magnanimo nunc sub Lemeingre Iohanne; Regius hic marescallus tua sceptra gubernat, Trans hominem solers et pacis cultor et equi: Ergo diu gaude sub tanto rege beata.

E' pure a lui attribuito l'epitaffio di Guido Septem, arcivescovo di Genova, inciso sul monumento funebre del medesimo a Portofino, mentre si suppone che gli siano dovute anche le iscrizioni sulla tomba degli Stella nel chiostro della chiesa di San Domenico. L'ultima opera poetica di lui nota e il componimento che egli invio a Pier Candido Decembrio tra il 1426 ed il 1428 "per esaltare l'operosità del cancelliere milanese ed il suo intervento, volto a migliorare le relazioni tra Genova e Milano"3.

Possediamo il suo epistolario, composto per dovere d'ufficio nella cancelleria genovese: degna di rilievo e la lettera da lui scritta a Carlo VI di Francia il 21 ottobre 1409 per accusare il governatore Boucicaut e difendere la condotta dei Genovesi, che si erano liberati del governo francese ${ }^{4}$.

L'opera cronachistica di Giovanni Stella è contrassegnata da una certa disorganicità, con notevoli lacune, tra cui appare rilevante quella relativa alle vicende dell'anno 1419 . Resta comunque interessante il fatto che egli tratta di vicende a cui egli stesso assistette o partecipd, si che il suo testo risulta attendibile ed importante, perché egli medesimo, in quanto cancelliere della Repubblica, fu a conoscenza di fatti interni dello Statos.

Anche nella cronaca di Giovanni Stella, come gia in quella del fratello Giorgio, resta tuttavia scarso l'interesse per il quadro delle "colonie" orientali della Repubblica, pure essendo il suo racconto ricco di dati sulle vicende interne dello Stato genovese. Ma l'Oriente appare lontano, immerso in una realtà che a Genova sembra spesso di non facile comprensione, nonostante lo stretto intreccio dei legami economici, intercorrenti tra quelle sedi e la madre-patria soprattutto grazie alle diramazioni familiari delle grandi casate genovesi. Comunque, uno dei centri di maggiore inte-

\footnotetext{
${ }^{3}$ Ibidem, pp. IV-V. I versi in ricordo dell'ampliamento di Castelletto sono riportati nella cronaca di Giorgio Stella, sotto l'anno 1402: ibidem, p. 256.

"Ibidem, p. V.

s'bidem, p. VI.
} 
resse del nostro cronista per il panorama orientale riguarda l'isola di Chio, affidata nel 1347 al governo della compagnia della Maona ${ }^{6}$.

Il primo accenno si riferisce al dicembre del 1408, quando nell'isola scoppio una rivolta contro il governo francese di Genova, al grido Vivat populus et Sanctus Georgius! Deposto il podestà e gli altri ufficiali, i Maonesi s'impadronirono delle merci di alcuni mercanti, stimate del valore di 15.000 ducati, per avere a disposizione dei capitali nel caso che $\mathrm{i}$ ribelli avessero a subire una spedizione punitiva e quindi dovessero provvedere alla difesa dell'isola. Ed infatti l'anno successivo il Boucicaut invio da Genova contro gl'insorti tre navi grosse al comando di Corrado Doria, il quale lascio Genova il 15 maggio. Raggiunta l'isola il 18 giugno, il Doria incontro una forte resistenza; pero, sia come sia, riuscl ad occupare la citta ${ }^{7}$.

Nel 1411 sono i Catalani a suscitare l'interesse del nostro cronista, sempre con riferimento all'isola di Chio. Egli scrive che in quell'anno, cum Ianuenses et Catalani exosi forent atque discordes, era giunta a Genova notizia certa che l'isola era stata attaccata, con lancio di sassi, da sette navi catalane per instrumenta, que vulgus bombardas nominat, ed inoltre che $i$ Catalani medesimi avevano pronunciato molte parole offensive contro l'onore dei Genovesi. L'onore per l'uomo medievale, ancorché fondato su presupposti non sempre coincidenti con il nostro odierno modo di vedere, e cosa molto importante: sicché i Genovesi, che si trovavano nell'isola per affari di commercio o che vi abitavano, mossi da non poca ira e per vendicare l'onore patrio, armarono per due mesi, con circa ottocento uomini, cinque navi mercantili, che erano nel porto. Il governo dei Maonesi dell'isola partecipd alle spese di armamento con 3.772 aurei, mentre il signore di Lesbo fornl un'altra galea armata. Il comando della flotta venne affidato alternativamente a Paolo Lercari ed a Battista de Franchis Luxardo, che erano diretti a Caffa per occupare colà, uno dopo l'altro, la carica di con-

\footnotetext{
${ }^{6}$ Sull isola di Chio nel secolo XIV cfr., fra l'altro, Philip P. ARGENTT, The occupation of Chios by the Genoese and their administration of the island (1346-1566), I, Cambridge, 1958; Geo PISTARINo, Nella "Romania" genovese tra i Greci e $i$ Turchi: l'isola di Chio, "Rivista Storica Italiana", LXIII-1 (1961), pp. 69-84; ID., Chio dei Genovesi, "Studi Medievali", 3 serie, X-1 (1969), pp. 3-68; Michel BALARD, La Romanie Génoise XIle - début du XVe siècle), Genova-Roma, 1978, passion; Laura BALLTTO, Chio dei Maonesi sulla fine del Trecento (dagli atti del notaio Donato di Chiavari), "Anuario de Estudios Medievales", 20 (1990), pp. 133-147.
}

'Georgii et Iohannis STELLAE cit., pp. 285-286, 288. 
sole del luogo. Il 18 luglio la flotta partl da Chio, il 20 approdo a Rodi, dove sosto brevemente per poi ripartire alla volta di Alessandria, dove si era saputo che i Catalani si erano rifugiati.

Le navi genovesi giunsero nel porto di Alessandria il successivo 24 luglio, verso l'ora del vespro. Vi entrarono per assalire le navi catalane; ma, essendo sopraggiunta la notte, gettarono le ancore e per tutta la notte restarono ferme presso le navi catalane. Anche il giorno successivo i Genovesi non presero alcuna iniziativa, studiando un piano d'azione, evidentemente per compensare la loro inferiorita numerica. Il 26 luglio, di domenica, le navi catalane si mossero contro le genovesi; le genovesi contro le catalane. Fu un duro scontro, con molti feriti da entrambe le parti. Il combattimento si protrasse fino a mezzogiorno, quando i Catalani, non parum exterriti, rapidamente si allontanarono. Tuttavia entrambe le nationes restarono per alcuni giorni nel porto, fronteggiandosi di continuo con le loro navi.

Un giorno i Catalani, vedendo che i Genovesi non prendevano nessuna iniziativa, mossero contro di loro, che erano quasi inermes, cioe non sembravano essere in assetto di guerra. Ma i Genovesi, ad uno squillo di tromba, del tutto inaspettatamente brandirono di colpo le armi, cosl che i Catalani, vedendo gli avversari tanto forti che essi non riuscivano ad infrangerne la resistenza, si allontanarono, catturarono una nave veneziana del tipo detto comunemente marrano, la riempirono di materiale combustibile e la scagliarono contro le navi genovesi, allo scopo di incendiarle. A questo punto i Genovesi, armati $\mathrm{i}$ cimbi $^{8}$, si preoccuparono di spingere lontano dalle loro navi il marrano, che dirottarono, cosl incendiato, verso terra. Vedendo inoltre che i Catalani avevano di riserva altre navi, e precisamente due marrani, si avvicinarono con i cimbi e li catturarono, togliendo cosi ai nemici la possibilità di mettere ancora a fuoco le navi genovesi.

Il giorno successivo i Genovesi, scaricato uno dei marrani, decisero di spedirlo in fiamme contro le navi catalane. Poiché avevano molti morti e feriti, disposero in assetto di combattimento le tre navi più grosse delle loro cinque, indirizzandole contro le navi catalane insieme con il marrano, pronto per appiccare l'incendio alle navi nemiche, se non si fossero arrese. Era circa l'ora del vespro quando, intimato l'ultimatum, lo squillo delle

\footnotetext{
${ }^{8}$ Si tratta di piccole imbarcazioni: Augustin JAL, Nouveau glossaire nautique, révision de l'édition publiée en 1848, Paris, 1978, alla voce cymba.
} 
trombe annunzio la battaglia. I Catalani, timentes, all'ora di compieta inviarono un messaggero con la proposta di restituire immediatamente alcune delle navi genovesi, che essi avevano catturato, purché la parte avversa desistesse dall'offensiva. I Genovesi rifiutarono decisamente: Nolumus: volumus enim cunctas naves et merces, quas a lanuensibus rapuerunt; et sic non offendemus eorumdem Catalanorum persona. Esigevano dunque la restituzione non soltanto di alcune, ma di tutte le navi a loro sottratte dai Catalani; ed anche la consegna delle merci, sebbene queste ultime non fossero di grande valore. Cosl infatti dichiara sinceramente il cronista, probabilmente per mettere in particolare evidenza la decisione genovese nel non transigere contro le sopraffazioni.

Il messaggero se ne ando, ritornando poi la notte stessa per riferire che i Catalani erano disposti ad accettare le richieste dei Genovesi: avrebbero restituito tutte le navi e le merci che avevano sottratto. Le due parti raggiunsero cosl un accordo. Ma nelle ultime ore della notte successiva, mentre i Genovesi montavano l'ultima veglia di guardia, i Catalani violentemente si agitarono -tanto da indurre i Genovesi a mettere in armi le navi-, ed all'alba del 5 agosto alzarono d'improvviso le vele su due navi,che pero non si poterono muovere perché toccavano il fondo del mare. Subito i Genovesi tolsero loro una nave grossa -che era genovese, ma era una di quelle catturate dai Catalani-, insieme con un'altra nave, anch'essa già genovese, e con una terza nave. Tutto cio fu condotto ad effetto grazie all'eroico comportamento di Battista de Franchis, il quale si mosse con un'azione pronta, strenua e decisa, combattendo audacemente: cosi deciso all'azione che volle egli stesso imbarcarsi con pochi compagni in una piccola scialuppa per dirigersi al porto, con grande pericolo, al fine di portare a compimento la progettata missione. A Paolo Lercari tocco una sorte più grave: mentre combatteva coraggiosamente fu colpito al volto da un colpo di balestra. Entrambi questi comandanti -commenta il cronista- sono degni di grande lode.

Due navi catalane erano dunque incagliate sul fondo del mare. Ed i Catalani, mentre i Genovesi erano intenti a predare le navi che avevano catturato, provvidero a scaricarle ed a trainarle a terra, sl che i Genovesi non potessero raggiungerle. Sulle tre navi tolte ai Catalani, i Genovesi fecero prigionieri circa cento uomini, sia catalani sia di altre nazioni; inoltre si impadronirono di un bottino di circa 10.000 aurei e liberarono i pochi genovesi che i nemici tenevano strettamente incatenati sulle navi. Le restanti delle sette navi dei Catalani erano state da questi vendute ai Mori. I 
Genovesi scaricarono poi una delle navi che avevano preso ai Catalani, trainandola presso le altre navi nemiche, insieme con il loro proprio marrano, allo scopo di dare il tutto alle fiamme. Ma non vi riuscirono, come avrebbero invece potuto fare in precedenza, quando non avevano voluto agire nella speranza di poter conservare le navi indenni insieme con le merci.

I Genovesi restarono per diversi giorni nel porto di Alessandria cercando il modo per sopraffare gli avversari. Una loro nave ed un marrano si trovavano vicini alle navi catalane e quindi in posizione distanziata dal resto della loro flotta. Essi, quanto mai fiduciosi delle loro forze, li avevano lasciati pressoché in disarmo. Ma, spirando un forte vento favorevole, $\mathrm{i}$ Catalani assalirono questi due vascelli, catturando coloro che erano saliti a bordo mentre tentavano di fuggire. Allora i Genovesi scaricarono le loro tre navi, portandole a terra presso le navi catalane. Si combatté per quindici giorni: i Genovesi tentarono di incendiare le navi catalane ed i Catalani tentarono di incendiare le navi genovesi: inutilmente dall'una e dall'altra parte.

A questo punto intervenne il governo di Alessandria, mosso da risentimento verso le due parti belligeranti, soprattutto contro i Genovesi, i quali inviarono un'ambasceria a terra per sedare la vertenza. Ma gli ambasciatori vennero arrestati e non poterono più fare ritorno. Allora i Genovesi, non potendo fare più nulla contro $\mathrm{i}$ nemici, dal momento che mancavano ormai di acqua e di viveri, bruciarono tutte le navi che avevano tolto ai Catalani -ad eccezione di una che si portarono via da Alessandria- ed il giorno 25 agosto partirono, lasciando le due navi catalane incagliate a terra con il loro carico di merci del valore di 80.000 aurei.

Quando le navi genovesi giunsero a Rodi, seppero che alcuni Catalani parlavano superbamente e contro la verità circa lo svolgimento dei fatti, ad esaltazione di loro medesimi ed a disonore dei Genovesi. Il cronista riferisce di avere saputo che quei Catalani che erano stati portati sulle navi genovesi furono impiccati ${ }^{9}$.

L'impresa genovese si era dunque risolta in un fallimento, e Giovanni Stella non lo nasconde. Ma perché -ci chiediamo- gli ha dato tanto risalto? C'e evidentemente, come gia detto, l'intenzione di porre in luce la capacità operativa delle navi genovesi, l'abilità dei comandanti, la perizia

${ }^{9}$ Georgii et lohannis STELLAE cit., pp. 304-306. 
degli equipaggi, il coraggio di tutti gli uomini della ciurma. Ma credo anche che debba vedersi nel rilievo dato a questo episodio un riflesso del tempo in cui scrive il nostro cronista: quando cioè la rivalità fra Genova e la Corona catalano-aragonese nel Mediterraneo occidentale si fa più aspra, sl che egli vuole mettere bene in evidenza contro quali nemici i suoi compatrioti devono lottare e quali sono le ragioni per cui Genova combatte contro ripetuti tentativi di sopraffazione che investono non soltanto l'Occidente ma anche le "colonie" del mondo orientale.

L'ampio spazio, dedicato da Giovanni Stella all'episodio dei corsari catalani, assai maggiore di quello riservato alla ribellione dei Maonesi contro il governo francese di Genova, presenta due aspetti. Uno e quello della minuzia dell'informazione sull'episodio stesso, la quale dimostra un'indubbia notizia di prima mano che il cronista dovette raccogliere presso qualche partecipe o testimone diretto della vicenda. L'altro riflette l'importanza attribuita dallo Stella all'episodio stesso, che egli certamente connette con la pressione esercitata al suo tempo dai Catalano-aragonesi nel Mediterraneo orientale e con la situazione di non pace e non guerra tra Genova e Barcellona.

La consistenza della stessa flotta catalana -sono sette navi- induce a ritenere che non si tratti di un episodio di pirateria, ma di vero e proprio corsarismo e che la sua organizzazione stessa sia stata messa in atto nel Mediterraneo occidentale, in una delle sedi catalane. E' evidente dunque che la Corona catalano-aragonese organizza la famosa "diagonale insulare" non soltanto con l'occupazione di aree territoriali -Sardegna, Sicilia-, ma anche cercando di colpire i punti nevralgici dell'Impero coloniale genovese in Oriente, quale era appunto anche l'isola di Chio. Evidentemente Giovanni Stella ha chiaro in mente tutto il quadro politico-militare e lo mette in risalto calcando le tinte sull'episodio che abbiamo riferito, nel quale, oltre a volere porre in luce la valentia genovese nelle armi, egli mette anche in evidenza la diffïcoltà delle imprese in questa lunga ed estenuante guerra marittima in cui talvolta i risultati non erano cosi brillanti come ci si attendeva e come rivela il nostro episodio.

Giovanni Stella vorrebbe evidenziare la superiorità genovese sull'altra grande rivale nel Mediterraneo: la marina catalana, che si e mossa e si sta muovendo per la conquista del mercato del Mediterraneo attraverso la "diagonale insulare". Il cronista vuole sottolineare le difficolta delle azioni a cosl forte distanza dal proprio mare, il Tirreno ed il Mediterraneo occidentale; ma si intuisce che, nel fondo del suo animo, ha trovato spazio il 
senso dell'avversione, destinata ad accrescersi in Genova, contro l'avanzata catalana nel Mediterraneo, la quale non si limita più al settore occidentale, ma si fa strada anche in quello orientale, com'era già clamorosamente avvenuto con la spedizione degli Almogaveri in Oriente, dopo la guerra del Vespro $^{10}$.

In occasione più rilevante Chio ritorna nelle pagine del nostro cronista con riferimento alla spedizione veneziana sull'isola del 1431 . Venezia, che prima aveva cercato inutilmente di conquistare Genova, aveva mosso il suo attacco contro le colonie genovesi nel Levante, in particolare contro Chio, che già la Serenissima riteneva giustamente essere il pilastro dell'apparato genovese nell'Egeo e nel Vicino Oriente. Giovanni Stella evidenzia la sconfitta subita dagli aggressori, che ebbero perdite rilevantissime, malgrado i Genovesi fossero soltanto in numero di trecento sotto la guida di Raffaele Montaldo. Né il cronista dimentica gli aiuti approntati a Genova per la difesa dell' isola (anche se commette un piccolo errore cronologico): tre navi grosse di Tommaso Squarciafico, di Filippo Giustiniani e di Gerolamo Falamonica, con millecinquecento uomini; più due galere, patronizzate da Martino de Nigrono e Pelegro Assereto. Le quali imbarcazioni furono inviate a Chio sotto il comando di Tommaso Scipiono Cebà. Partirono da Genova verso la fine di dicembre, ma giunsero nell'isola il 25 marzo 1432.

Dovette essere una navigazione assai lenta, sia per causa del tonnellaggio delle navi grosse, sia per causa del tempo invernale, che non offriva molte ore di luce. Comunque, quando la flotta genovese giunse a Chio, il problema si era ormai risolto: i veneziani avevano già sgombrato il campo $^{11}$. La mossa veneziana aveva pero mostrato quanto fosse precaria la situazione delle "colonie" orientali, al cui soccorso la madre-patria poteva provvedere con estrema lentezza, mentre Venezia disponeva di basi di appoggio assai più prossime (basta pensare a Candia ed all'Eubea) e per di più si trovava ad assai minore distanza dal Vicino Oriente.

\footnotetext{
${ }^{10}$ Sugli Almugaveri in Oriente cfr., fra l'altro, Giustina OLGIATT, Ramon Muntaner $e$ l'"expedició" dels Calalans a Orient, "Saggi e Documenti Vl", Civico Istituto Colombiano, Studi e Testi. Seric storica, 8, Genova, 1985, pp. 207-266; Geo PISTARINO, L'Impero greco tra Genovesi ed Alinugaveri, in Geo PISTARINO, I Signori del mare, Civico Istituto Colombiano, Studi e Testi. Seric storica, 15, Genova, 1992, pp. 155-206.

"Georgii el lohannis STELAE, cit., pp. 373-374. Sulla spedizione veneziana a Chio cfr. Philip P. ARGENT, cit., pp. 176- 182.
} 
Questi, ricordati da Giovanni Stella, sono tre episodi di natura evenemenziale assai diversa tra l'uno e l'altro, ed in apparenza di diversa valenza storica. Il primo -quello della ribellone dei Maonesi di Chio al governo francese su Genova- rientra nel quadro della storia stessa della capitale ligure. Quello dell'incursione dei corsari catalani ci si presenta come un episodio di guerriglia sul mare, una guerriglia persistente. Quello dell'attacco veneziano a Chio appare anch'esso come un episodio della costante rivalita tra le due grandi repubbliche marinare italiane. In realtà sono tre momenti di uguale valore nel grande quadro della storia del primo Quattrocento genovese. Fanno parte, cioe, del medesimo panorama euro-mediterraneo, e dimostrano come nel mondo coloniale genovese del Vicino Oriente si riflettessero con estrema incidenza sul caposaldo di Chio gli avvenimenti più importanti della situazione interna e della posizione internazionale della Repubblica.

Giovanni Stella merita un riconoscimento: si dimostra uno scaltrito interprete delle vicende, sia prossime sia lontane, nella loro totale valenza storica. Quando, ad esempio, come nel nostro caso, egli accenna a Chio, ciò non avviene per semplice curiosità narrativa occasionale, ma per uno scopo preciso: l'attenta valutazione dello scrittore circa l'importanza degli elementi che egli inserisce nella sua narrazione.

La quale si conclude, sempre per riguardo a Chio, con un'ultima breve notizia: la spedizione di Carlo Lomellini, che nel marzo del 1434 partl da Genova diretta versus partes orientales: Chio, Mitilene, Pera, Caffa, con un totale di seimila uomini' ${ }^{12}$. E' l'ultimo accenno, ma quanto basta per completare il quadro, tenuto anche conto che la cronaca termina con il 1435. E tuttavia, vista nelle dimensioni del tempo, si trattava di una impresa di cui il cronista non specifica la finalità, trattandosi, come sempre in Genova, della ben nota "politica del segreto", anche se rappresenta una delle ultime grandi iniziative politico-militari da parte genovese per cercare di tenere sotto controllo l'impero nel Levante, che e avviato ad un rapido tramonto. Ma essa non suscita la fantasia dello scrittore, sia perché forse non ebbe modo di conoscerne i particolari, sia perché vi mancarono gli elementi che potevano offrire colorito alla narrazione, come invece fu per lui l'episodio dell'impresa catalana su Chio.

${ }^{12}$ Georgii et Iohannis STFIJAE, cit., p. 378. 
Come dimostra attraverso la sua attività poetica, seppure non eccelsa, Giovanni Stella è autore di viva sensibilità di fronte agli eventi umani. La scelta dei temi della sua cronaca risponde -nei casi specifici, di cui ci siamo occupati in questa breve nota- a quella che era in lui la visione del panorama del tempo, e rappresenta per noi, data la sua più o meno riconosciuta oggettività di giudizio, un contributo valido per determinare la prospettiva di valutazione sulle vicende del passato.

\section{RÉSUMÉ}

Giovanni Stella, le plus grand chroniqueur génois du XVe siècle, dont la chronique raconte les événements de 1408 a 1435 , ne fait pas souvent allusion à la présence génoise dans le Proche-Orient. Cependant, dans trois passages de la chronique, il montre une remarquable capacité d'interprétation des événements génois dans la Méditerranée orientale, au-delà de la simple curiosité narrative occasionelle et une évaluation attentive de l'importance historique des événements mêmes. Ces passages de la chronique de Giovanni Stella font référence à l'île de Chio, qu'il considère justement comme le centre le plus important de la présence génoise dans le Proche-Orient. Il s'agit de la révolte des Mahonais de Chio en 1408; d'une expédition catalane contre l'île de Chio en 1411 et de la contre-offensive des navires génois qui poursuivent les navires catalans jusqu'à Alexandrie de Syrie sans un grand résultat positif; d'une expédition vénitienne contre l'île de Chio en 1431.

\section{SUMMARY}

Giovanni Stella, the most important XVth Century genoese chronicler, whose chronicle relates the events from 1408 to 1435 , hardly ever makes any reference to the genoese Levant. Nevertheless, in three of the passages of his chronicle, he demonstrates a high capacity of interpretation of the genoese events in the Eastern Mediterranean. These passages are much more than a mere account of facts, because the author offers us even 
an attentive evaluation of the events. The Giovanni Stella's Chronicle passages are referred to the isle of Chios -that is considered by Stella as the most important centre of the genoese presence in the Levant-, and deal on the following subjects: the revolution of the "maonesi" of Chios in 1408; a catalane expedition against Chios in 1411, followed by the genoese counteroffensive that pursued the catalan ships to Alexandria (Syria), without great success; and, finally, a venetian expedition against Chios in 1431. 